Article

\title{
Involvement of FvSet1 in Fumonisin B1 Biosynthesis, Vegetative Growth, Fungal Virulence, and Environmental Stress Responses in Fusarium verticillioides
}

\author{
Qin Gu ${ }^{\dagger}$, Hafiz Abdul Samad Tahir ${ }^{\dagger}$, Hao Zhang, Hai Huang, Tiantian Ji, Xiao Sun, Liming Wu, \\ Huijun $\mathrm{Wu}^{*}$ and Xuewen Gao * \\ Key Laboratory of Monitoring and Management of Crop Diseases and Pest Insects, \\ Department of Plant Pathology, College of Plant Protection, Nanjing Agricultural University, \\ Ministry of Education, Nanjing 210095, China; guqin@njau.edu.cn (Q.G.); 2014202054@njau.edu.cn (H.A.S.T.); \\ 2014102015@njau.edu.cn (H.Z.); huanghai_201510@163.com (H.H.); 2015102015@njau.edu.cn (T.J.); \\ 2016102012@njau.edu.cn (X.S.); wuliming@njau.edu.cn (L.W.) \\ * Correspondence: hjwu@njau.edu.cn (H.W.); gaoxw@njau.edu.cn (X.G.); Tel.: +86-25-8439-5268 (H.W.); \\ $+86-25-8439-5268$ (X.G.) \\ + These author contributed equally to this work.
}

Academic Editors: Susanne Vogelgsang and Yang Liu

Received: 14 December 2016; Accepted: 17 January 2017; Published: 24 January 2017

\begin{abstract}
Fusarium verticillioides (teleomorph, Gibberella moniliformis) is an important plant pathogen that causes seedling blight, stalk rot, and ear rot in maize (Zea mays). During infection, F. verticillioides produce fumonsins B1 (FB1) that pose a serious threat to human and animal health. Recent studies showed that Set1, a methyltransferase of H3K4, was responsible for toxin biosynthesis in filamentous fungi. However, to date, the regulation of FvSet1 on FB1 biosynthesis remains unclear. In the current study, we identified only one Set1 ortholog in F. verticillioides (FvSet1) and found that the deletion of FvSET1 led to various defects in fungal growth and pathogenicity. More interestingly, the FvSET1 deletion mutant ( $\triangle$ FvSet1) showed a significant defect in FB1 biosynthesis and lower expression levels of FUM genes. FvSet1 was also found to play an important role in the responses of $F$. verticillioides to multiple environmental stresses via regulating the phosphorylation of FvMgv1 and FvHog1. Taken together, these results indicate that FvSet1 plays essential roles in the regulation of FB1 biosynthesis, fungal growth and virulence, as well as various stress responses in F. verticillioides.
\end{abstract}

Keywords: Fusarium verticillioides; FvSet1; fumonisin B1; fungal growth and virulence; stress responses

\section{Introduction}

Fusarium verticillioides (teleomorph, Gibberella moniliformis) is an important plant pathogen that causes seedling blight, stalk rot, and ear rot in maize (Zea mays), which is one of the most common diseases of affecting maize worldwide [1,2]. More importantly, during growth in maize, F. verticillioides produces various mycotoxins, such as fumonisins, which are extremely harmful to human and animal health [3,4]. Fumonisins have been shown to play critical roles in the development of maize diseases caused by F. verticillioides [5]. Among the diverse forms of the fumonisins, fumonisins B1 (FB1) is the most prevalent and well-characterized. A cluster of 17 FUM genes is known to be responsible for FB1 biosynthesis [6,7]. However, the molecular mechanisms underlying the regulation of FUM gene transcription in F. verticillioides remains to be elucidated.

In eukaryotic cells, regulation of gene transcription is associated with multiple modifications of histones such as methylation, phosphorylation, adenosine diphosphate (ADP)-ribosylation, 
biotinylation, acetylation, and ubiquitination [8]. Among these modifications, histone H3 lysine 4 methylation (H3K4me) was found to activate gene transcription in various organisms, including yeast, plants, and animals [9-12]. Recent studies have shown that methylation of lysine 4 on histone H3 has important influences on secondary metabolism and fungal virulence in several pathogenic fungi, such as Fusarium graminearum and Magnaporthe oryzae. In F. graminearum, H3K4me plays important roles in deoxynivalenol (DON) production by positively regulating transcription of TRI genes, which encode trichothecene biosynthetic enzymes and regulators [13]. A global view of the relationship between $\mathrm{H} 3 \mathrm{~K} 4 \mathrm{me}$ and gene transcription in $\mathrm{M}$. oryzae obtained by CHIP-seq analysis revealed an association between H3K4me and activation of gene expression and regulation of fungal virulence [14].

In Saccharomyces cerevisiae, the Set1/COMPASS complex, consisting of Set1 and its subunits, functions as a methyltransferase capable of catalyzing the mono-, di-, and trimethylation of H3K4 [15-17]. Deletion of S. cerevisiae Set1 resulted in transcriptional silencing and growth defects [18]. Similar to $S$. cerevisiae, Set1 is essential for H3K4me in diverse pathogenic fungi, including F. graminearum and M. oryzae. Disruption of SET1 also leads to abnormal secondary metabolism and defects in fungal virulence [13,14]. In addition, a recent study in F. graminearum revealed that FgSet1 is associated with multiple stress responses that extend our understanding of the functions of H3K4 methylation on environmental stress responses in filamentous fungi [13].

In this study, we identified the FvSET1, which encodes H3K4 methyltransferase. For genetic characterization of FvSet1, we generated the FvSET1 deletion mutant. Phenotypic analysis showed that FvSet1 plays critical roles in FB1 biosynthesis, fungal growth and virulence, and stress responses in F. verticillioides. Moreover, in the current study, it was found that FvSet1 is required for activation of FUM gene expression. These findings provide a clearer understanding of regulation of FB1 biosynthesis in F. verticillioides, which will be beneficial to establishing the efficient strategies for FB1 contamination management.

\section{Results}

\subsection{In Silico Analysis of FvSet1 in F. verticillioides}

To elucidate the function of $\mathrm{H} 3 \mathrm{~K} 4 \mathrm{me}$ in F. verticillioides, we searched for the ortholog of S. cerevisiae Set1, which is the core component of methyltransferase complex Set1/COMPASS. Using S. cerevisiae Set1 as a query, we found only one Set1 ortholog, FVEG_07811 (designated FvSET1), from the F. verticillioides genome via BLASTP. FvSET1 was $3901 \mathrm{bp}$ in length and interrupted with three introns. FvSet1 was predicted to encode a 1234 amino acids protein and harbor two typical SET domains. The putative amino acids sequences of FvSet1 shared 56\% identity with that of yeast Set1 (Figure S1A). Additionally, phylogenic analysis showed that the FvSet1 was highly homologous to its counterparts in yeast and other pathogenic fungi (Figure S1B).

\subsection{Deletion and Complementation of FvSet1 in F. verticillioides}

To characterize the function of FvSet1, we generated a FvSET1 deletion mutant ( $\triangle$ FvSet1) using a homologous recombination strategy (Figure S2A). Among 28 transformants, three FvSET1 deletion mutants were identified by polymerase chain reaction (PCR) analysis with the primer pair A5 + A6 (Table S1). Southern blot analysis confirmed that $\Delta$ FvSet1 resulted from the anticipated homologous recombination of FvSET1 (Figure S2B). To confirm that various defects in $\triangle$ FvSet1 were the result of FvSET1 deletion, a full-length FvSET1 was transformed into the $\triangle$ FvSet1 deletion mutant and the resulting complementation strain $\triangle$ FvSet1-C was identified by PCR analysis using the A5 + A6 primer pairs.

\subsection{FvSet1 Is Involved in Fungal Growth and Conidial Germination of F. verticillioides}

To characterize the function of FvSet1 in mycelial growth, each strain was cultured at $25^{\circ} \mathrm{C}$ on potato dextrose agar (PDA), complete medium (CM), and minimal medium (MM). After four days, $\Delta$ FvSet1 displayed a significant decreased growth rate (Figure 1). 


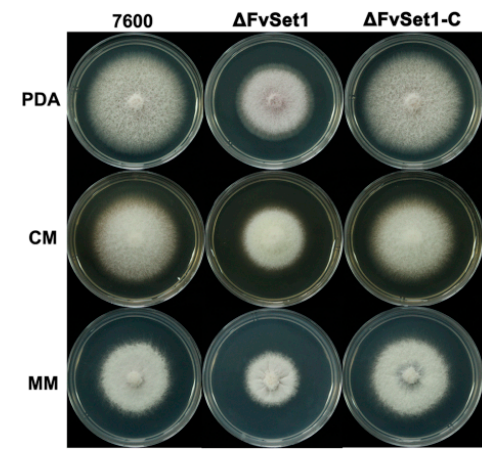

(A)

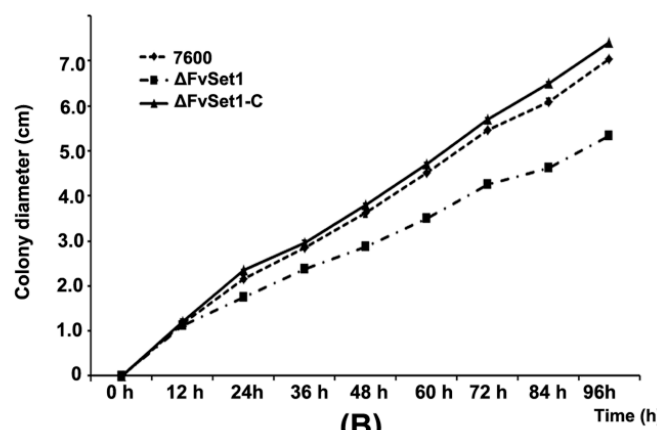

(B)

Figure 1. Impacts of FvSET1 deletion on F. verticillioides mycelial growth. (A) the wild-type (strain 7600), the FvSET1 deletion mutant ( $\triangle$ FvSet1), and the complementation strains $(\triangle \mathrm{FvSet1}-\mathrm{C})$ were cultured on PDA (Potato Dextrose Agar), CM (Complete Medium) and MM (Minimal Medium) at $25^{\circ} \mathrm{C}$ for four days; and (B) comparison of mycelial growth rates among the wild-type, $\Delta$ FvSet1, and $\Delta$ FvSet1-C on PDA medium. Line bars in each column denote standard errors of three repeated experiments.

To detect the roles of FvSet1 in asexual development, fresh mycelia $(50 \mathrm{mg})$ of each strain were cultured in liquid CMC medium at $25{ }^{\circ} \mathrm{C}$ in a shaking incubator. After four days, $\triangle \mathrm{FvSet} 1 \mathrm{did}$ not exhibit any significant defects in conidiation (data not shown). To determine the effect of FvSET1 deletion on conidal germination, conidia from each strain was used to inoculate in YEPD (Yeast Extract Peptone Dextrose) liquid medium and cultured at $25^{\circ} \mathrm{C}$ for $4 \mathrm{~h}$ and $6 \mathrm{~h}$. It was found that $\Delta \mathrm{FvSet} 1$ showed a similar percentage of conidial germination compared with that of the wild-type 7600 strain and the $\Delta$ FvSet1-C complementation strain (data not shown). However, the average length of germinated conidial germ tubes of the mutant was dramatically reduced when compared with those of the wild-type 7600 after incubation for $4 \mathrm{~h}$ and $6 \mathrm{~h}$ (Figure 2).

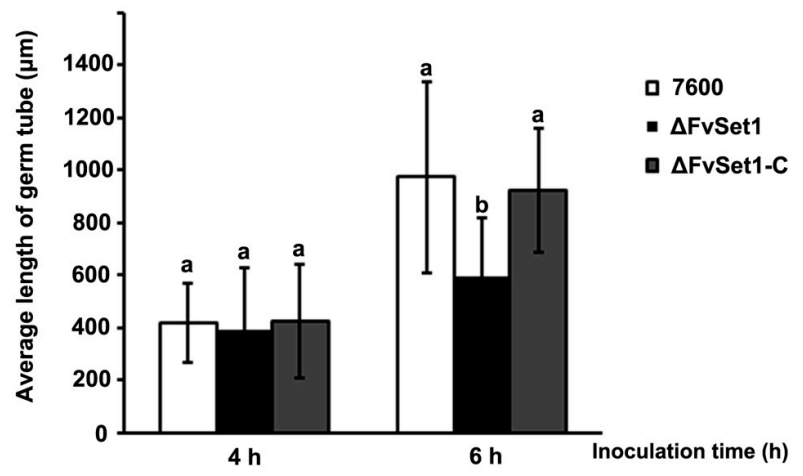

Figure 2. Involvement of FvSet1 in conidial germination in F. verticillioides. Average length of 150 germ tubes from each strain after incubation for $4 \mathrm{~h}$ and $6 \mathrm{~h}$ of in YEPD (Yeast Extract Peptone Dextrose Medium). Bars denote standard errors from three repeated experiments.

\subsection{FvSet1 Is Indispensable for Full Virulence in F. verticillioides}

To test the pathogenicity of each strain, we injected the conidia of the wild-type $7600, \Delta$ FvSet1, and $\Delta$ FvSet1-C into maize stalks. After 15 days, the typical symptoms of rot caused by $\Delta$ FvSet1 were significantly reduced when compared with the effects of the wild-type 7600 and the $\Delta$ FvSet1-C complementation strain on maize stalks under the same condition (Figure 3A,B). To investigate the roles of FvSet1 on colonization, damaged maize kernels were sterilized and then inoculated with a $10-\mu \mathrm{L}$ aliquot of conidial suspension of each strain. After seven days, a dramatic reduction inaerial hyphae in $\triangle$ FvSet1 was observed during pathogenesis compared with those of the wild-type and $\triangle$ FvSet1-C (Figure $3 C$ ). Furthermore, we examined the amount of fungal ergosterol in the inoculated 
maize kernels. As indicated in Figure 3D, a four-fold reduction in the amount of ergosterol produced by $\Delta$ FvSet1 was observed compared with that produced by wild-type and $\Delta \mathrm{FvSet} 1-\mathrm{C}$. These results strongly suggested that FvSet1 plays a critical role in virulence of F. verticillioides on maize.

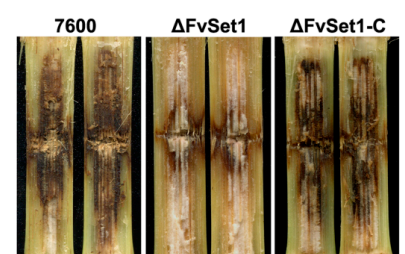

(A)

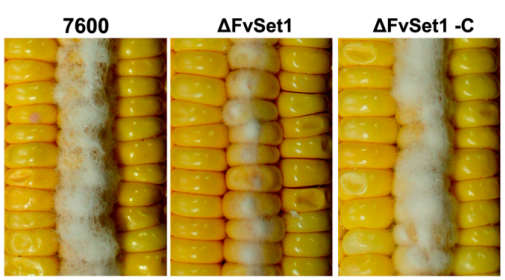

(C)

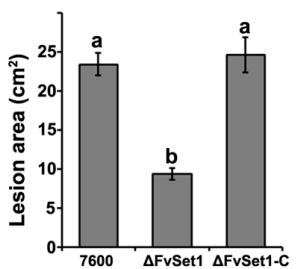

(B)

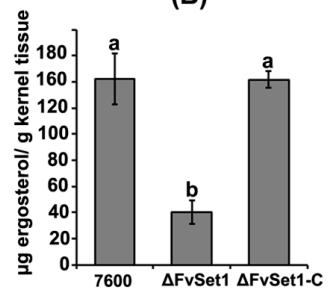

(D)

Figure 3. FvSet1 is required for full virulence in F. verticillioides. (A) maize stalk infection assays. Maize stalks were injected with conidial suspensions of the wild-type (Strain 7600), the FvSET1 deletion mutant $(\triangle \mathrm{FvSet} 1)$, and the complementation strain $(\triangle \mathrm{FvSet1}-\mathrm{C})$. Longitudinal dissections of infected maize stalks were photographed 15 days after inoculation; (B) lesion area of longitudinally dissected maize stalks infected with each strain for 15 days; (C) the wounded maize kernels were inoculated with conidial suspension of wild-type, $\Delta$ FvSet1, and $\Delta$ FvSet1-C. Infected maize kernels were photographed 15 days after inoculation; and (D) growth of the wild-type, $\Delta \mathrm{FvSet1}$, and $\Delta$ FvSet1-C strains on living maize kernels was evaluated by analysis of ergosterol biosynthesis. Values represent the mean ergosterol concentration in eight maize kernels sampled in triplicate from three separate ears for each strain. Line bars in each column denote standard errors of three repeated experiments.

\subsection{FvSet1 Is Required for FB1 Biosynthesis in F. verticillioides}

To determine the function of FvSet1 in FB1 biosynthesis, we analyzed the FB1 production in cracked maize kernels inoculated by wild-type, $\Delta$ FvSet1, and $\Delta$ FvSet1-C. At 21 days post-inoculation, the amounts of FB1 produced by the wild-type and complementation strains were 10-fold higher than that produced by $\Delta$ FvSet1 (Figure 4). Additionally, the FUM gene expression levels in $\Delta$ FvSet1 were significantly reduced compared with that in the wild-type following inoculation of liquid GYAM (Figure S3). These results showed that FvSet1 plays an important role in FB1 production by F. verticillioides via regulation of FUM gene transcription.

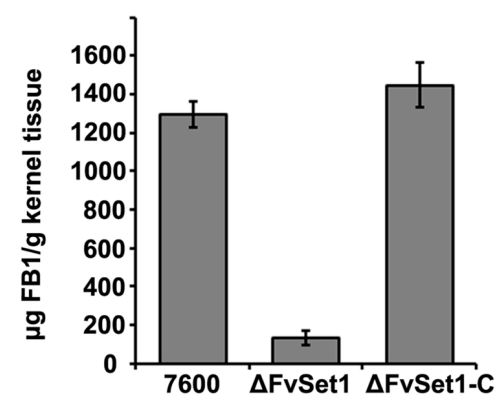

Figure 4. Effects of FvSET1 deletion on FB1 biosynthesis in F. verticillioides. The amounts of FB1 produced by each strain in infected maize kernels 21 days after inoculation. Line bars in each column denote standard errors of three replicated experiments. 


\subsection{FvSet1 Is Involved in Responses to Multiple Stresses Response}

To characterize the functions of FvSet1 in multiple stress responses, we tested the sensitivity of wild-type, $\Delta$ FvSet1, and $\Delta$ FvSet1-C to various environmental stresses. As indicated in Figure 5, $\Delta$ FvSet1 displayed significant increased sensitivity to osmotic stresses ( $0.7 \mathrm{M} \mathrm{NaCl}$ or $1.0 \mathrm{M}$ sorbitol) and oxidative stress [0.02\% SDS (Sodium dodecyl sulfate)] compared with the wild-type and the complementation strain. However, $\triangle$ FvSet1 exhibited dramatically increased resistance to a cell wall-damaging agent $(0.02 \%$ Congo red) compared with that of the wild-type and $\Delta$ FvSet1-C (Figure 5). In S. cerevisiae, the mitogen-activated protein kinase Mpk1 and Hog1 phosphorylation are required for maintenance of cell wall integrity and response to osmotic stresses, respectively. Thus, in this study, we also analyzed phosphorylation of FvMgv1 (an ortholog of S. cerevisiae Mpk1) and FvHog1 (an ortholog of S. cerevisiae Hog1) in F. verticillioides. As indicated in Figure 6A, the level of FvMgv1 phosphorylation in $\Delta$ FvSet1 was dramatically higher than that in the wild-type and the complementation strain $\triangle$ FvSet1-C. However, a decreased level of FvHog1 phosphorylation was observed in $\Delta$ FvSet1 when compared with that in the wild-type and $\Delta$ FvSet1-C (Figure $6 \mathrm{~B}$ ). These results indicated that FvSet1 play an important role in response of multiple environmental stresses by regulating the phosphorylation of FvMgv1 and FvHog1.

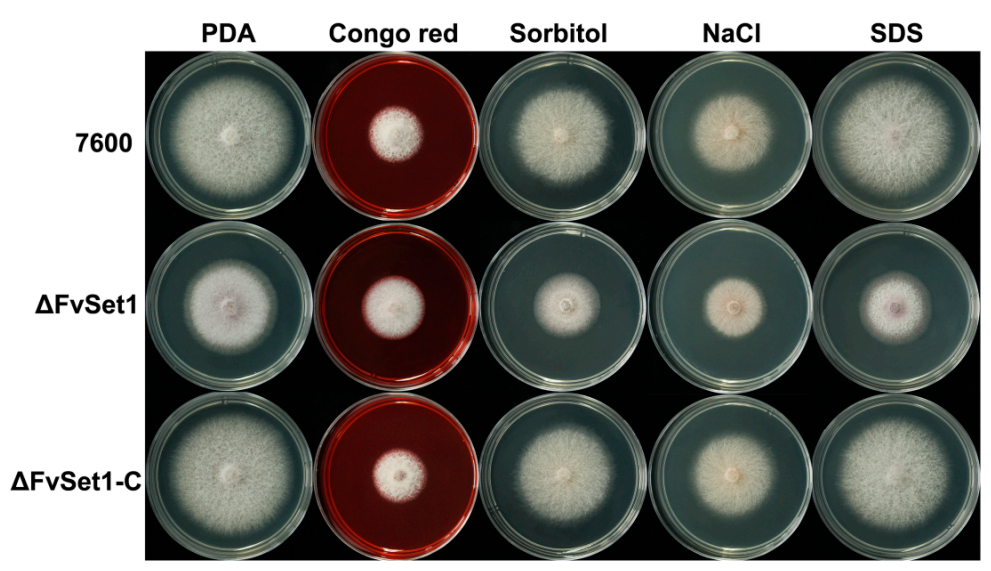

(A)

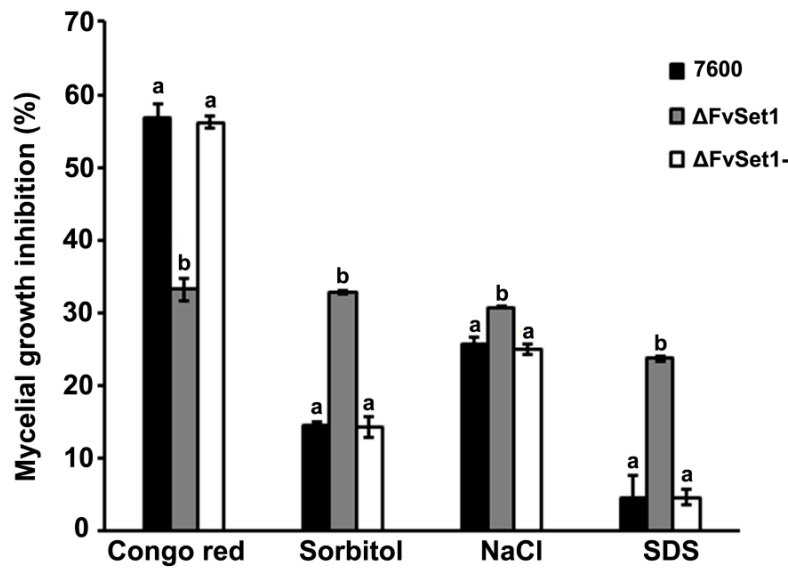

(B)

Figure 5. Sensitivity of the wild-type strain of F. verticillioides (Strain 7600), the FvSET1 deletion mutant $(\triangle$ FvSet1), and the complementation strain $(\triangle$ FvSet1-C) to osmotic and oxidative stresses, and cell wall-damaging agents. (A) Comparisons were made on PDA (Potato Dextrose Agar) with or without $0.02 \%(w / v)$ congo red, $1 \mathrm{M}$ sorbitol, $0.7 \mathrm{M} \mathrm{NaCl}$, and $0.02 \%(w / v)$ SDS (Sodium dodecyl sulfate); and (B) mycelial growth inhibition was examined after each strain was incubated for three days on PDA supplemented with each compound. Bars denote standard errors from three experiments. 


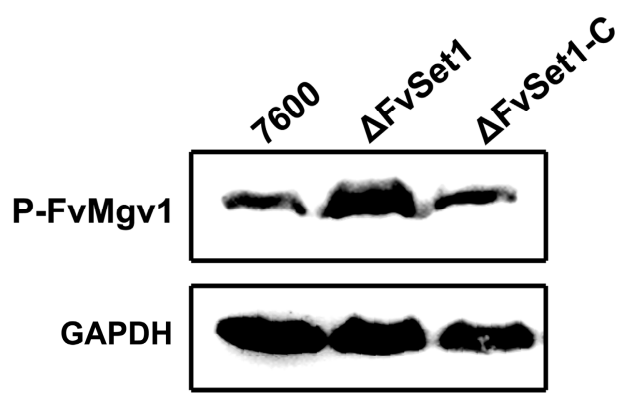

(A)

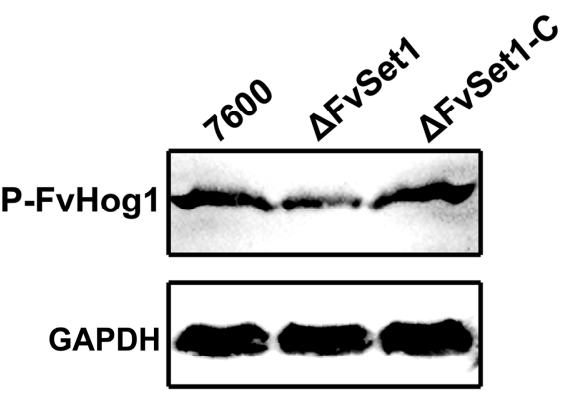

(B)

Figure 6. Involvement of FvSet1 in FvMgv1 and FvHog1 phosphorylation. Comparisons of phosphorylated FvMgv1 (A) and FvHog1 (B) in the wild-type of F. verticillioides (strain 7600), the FvSET1 deletion mutant $(\triangle \mathrm{FvSet})$ and the complementation strain $(\Delta \mathrm{FvSet1}-\mathrm{C})$. Phosphorylated FvMgv1 (P-FvMgv1) and FvHog1 (P-FvHog1) proteins were detected using the phospho-p44/42 and phospho-p38 MAP kinase antibody, respectively.

\section{Discussion}

Methylation of $\mathrm{H} 3 \mathrm{~K} 4$ is generally associated with transcriptional activation in eukaryotic cells $[19,20]$. Set1, the core component of methyltransferase Set1/COMPASS, was responsible for catalyzing the mono-, di-, and trimethylation of H3K4, which is highly conserved from yeast to humans [21]. Recently, Set1 was reported to play important roles in fungal growth, virulence, and secondary metabolism in several pathogenic fungi. However, the contribution of Set1 in F. verticillioides remains to be defined. Thus, in this study, we investigated the roles of FvSet1 on mycotoxin biosynthesis, fungal virulence, and fungal growth in F. verticillioides.

Recent studies show that the expression of the secondary metabolite cluster is associated with H3K4me [22]. In Aspergillus nidulans, CclA, which is a member of the Set1/COMPASS complex, plays important roles in di- and trimethylation of H3K4. The CCLA deletion mutant showed increased production of several secondary metabolites [23]. In contrast to its roles as a negative regulator of secondary metabosim in A. nidulans, FgSet1 positively regulates secondary metabolism in F. graminearum. Deletion of FgSET1 is associated with dramatic defects in DON and aurofusarin biosynthesis. In F. verticillioides, the biochemical pathway for fumonisin biosynthesis is well established. The FUM gene cluster consists of 17 FUM genes at a single locus and is responsible for encoding FB1 biosynthetic enzymes and regulators [6]. However, to date, the regulation of FUM gene cluster is relatively unclear. In this study, we found a dramatic reduction of FB1 production in $\triangle F v S e t 1$ compared with that in the wild-type and complementation strain, which is in accordance with what is known in F. graminearum. (Figure 4). Furthermore, FUM gene expression decreased significantly in $\triangle F v S e t 1$, indicating that FvSet1 positively regulates FB1 biosynthesis at the level of FUM gene transcription (Figure S3). Taken together, these results showed that functions of H3K4me in regulation of secondary metabolism vary significantly in different eukaryotes.

In S. cerevisiae, Set1 null mutants exhibited morphological, developmental and growth defects [24]. Similarly, FvSet1 was shown to be associated with fungal growth and virulence in F. graminearum and M. oryzae $[13,14]$. In the current study, FvSET1 deletion mutants displayed similar defects in fungal growth and conidial germination (Figures 1 and 2). More importantly, pathogenicity tests showed that $\Delta$ FvSet1 exhibited significantly reduced virulence in maize stalks and kernels, suggesting that FvSet1 is essential for fungal virulence in F. verticillioides (Figure 3). These results suggest that FvSet1 play similar roles in vegetative growth and the pathogenesis of pathogenic fungi.

To date, information regarding the relationship between H3K4me and environmental stress responses is scarce. Thus, in this study, we conducted sensitivity assays of various environmental stresses including osmotic, oxidative, and cell wall stresses. FvSET1 deletion led to increased sensitivity to osmotic stresses ( $\mathrm{NaCl}$ or sorbitol) and oxidative stress (SDS), while resistance to the 
cell wall-damaging agent (congo red) was significantly increased (Figure 5). Consistent with this finding, $\Delta$ FvSet1 showed a higher level of FvMgv1 phosphorylation and a decreased level of FvHog1 phosphorylation in comparison with those in the wild-type and the complementation strain (Figure 6). However, in F. graminearum, $\Delta$ FgSet1 did not exhibit a detectable change in sensitivity to osmotic and oxidative stresses [13]. Taken together, these results indicated that FvSet1 play a distinct role in response to multiple environmental stresses in F. verticillioides.

\section{Materials and Methods}

\subsection{Fungal Strains, Growth Conditions and Sporulation Tests}

For the wild-type strain of F. verticillioides strain 7600, the deletion mutants derived from the wild-type and the complementation strains used in our study were cultured at $25{ }^{\circ} \mathrm{C}$ on complete medium (CM) agar (10 g glucose, $2 \mathrm{~g}$ peptone, $1 \mathrm{~g}$ yeast extract, $1 \mathrm{~g}$ casamino acids, nitrate salts, trace elements, 0.01\% vitamins, $10 \mathrm{~g}$ agar and $1 \mathrm{~L}$ water, $\mathrm{pH}$ 6.5) [25], potato dextrose agar (PDA) (200 g potato, $20 \mathrm{~g}$ glucose, $10 \mathrm{~g}$ agar and $1 \mathrm{~L}$ water), and minimal medium (MM) agar $\left(10 \mathrm{mM} \mathrm{K}_{2} \mathrm{HPO}_{4}\right.$, $10 \mathrm{mM} \mathrm{KH}_{2} \mathrm{PO}_{4}, 4 \mathrm{mM}\left(\mathrm{NH}_{4}\right)_{2} \mathrm{SO}_{4}, 2.5 \mathrm{mM} \mathrm{NaCl}, 2 \mathrm{mM} \mathrm{MgSO}_{4}, 0.45 \mathrm{mM} \mathrm{CaCl}_{2}, 9 \mathrm{mM} \mathrm{FeSO}, 10 \mathrm{mM}$ glucose, $1 \%$ agar, $\mathrm{pH} 6.9$ ) for mycelial growth tests.

To characterize the conidiation of each strain, we added $50 \mathrm{mg}$ mycelia into a 50-mL flask

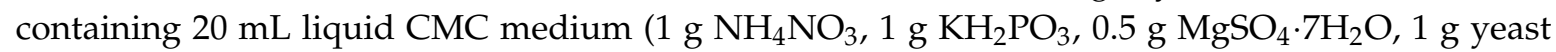
extract, $15 \mathrm{~g} \mathrm{CMC}$ and $1 \mathrm{~L}$ water). The flasks were incubated at $25^{\circ} \mathrm{C}$ for 4 days in a shaker (180 rpm). Conidia were counted by using a hemacytometer (Baili, Shanghai, China). The experiment was repeated on three independent occasions.

\subsection{Constructed Gene Deletion and Complemented Strains}

The FvSET1 replacement construct was generated using the double-joint PCR approach. The constructs were transformed into the protoplasts of F. verticillioides using the protocols described in previous described protocols [26]. The primers used to amplify the sequences upstream and downstream of FvSET1 are listed in Table S1. Putative mutants were identified by PCR using the relevant primers (Table S1), and were confirmed by Southern blotting assay (Figure 2B). All the strains used in this study were preserved in $30 \%$ glycerol at $-70{ }^{\circ} \mathrm{C}$.

\subsection{Pathogenicity Assays}

To test the pathogenicity of wild-type, deletion mutant $(\Delta$ FvSet 1$)$, and the complementation $(\triangle \mathrm{FvSet1-C})$ strain on maize stalks, conidia of each strain were collected by filtration through three layers of cotton gauze and subsequently re-suspended in sterilized water at $10^{6}$ conidia/mL. A sample of the conidial suspension $(10 \mu \mathrm{L})$ was used to inoculate maize cultivar B73 via a hole punched in the stem. The control maize stalks were inoculated with $10-\mu \mathrm{L}$ of sterilized water. Six replicates were prepared for each strain. After inoculation, the maize stalks were maintained at $25 \pm 2{ }^{\circ} \mathrm{C}$ under $80 \%$ humidity for 15 days. Fifteen days after inoculation, the infected maize plants were recorded. The experiment was repeated on four independent occasions.

To examine ability of each strain to infect maize kernels, a $10-\mu \mathrm{L}$ aliquot of a conidial suspension was used to inoculate the damaged maize kernels after surface sterilization. Five replicates were prepared for each strain. Maize kernels were incubated at $25^{\circ} \mathrm{C}$ under $100 \%$ humidity with a $12 \mathrm{~h}$ of daylight period, and were photographed 7 days after inoculation. The experiment was repeated on three independent occasions.

\subsection{Determination of FB1 Production}

A 25-g aliquot of healthy maize kernels was sterilized and inoculated with five mycelial plugs of each strain. After incubation at $25{ }^{\circ} \mathrm{C}$ for 21 days, fumonisin B1 was assayed as described previously $[27,28]$. The amount of FB1 andergosterol in each sample was determined using a HPLC 
system Waters 1525 (Waters, Milford, MA, USA) [29]. The experiment was repeated three times, and data were analyzed by analysis of variance (ANOVA; SAS version 8.0; SAS Institute, Cary, NC, USA).

\subsection{RNA Extraction and Quantitative Real-Time PCR}

For total RNA extraction, mycelia of each strain were used to inoculate liquid GYAM (0.24 M glucose, $0.05 \%$ yeast extract, $8 \mathrm{mM}$ L-asparagine, $5 \mathrm{mM}$ malic acid, $1.7 \mathrm{mM} \mathrm{NaCl}, 4.4 \mathrm{mM} \mathrm{K}_{2} \mathrm{HPO}_{4}$, $2 \mathrm{mM} \mathrm{MgSO}_{4}$, and $8.8 \mathrm{mM} \mathrm{CaCl}_{2}, \mathrm{pH} 3.0$ ) or liquid $\mathrm{CM}$ for $36 \mathrm{~h}$ at $25^{\circ} \mathrm{C}$ in the shaking incubator (200 rpm). Mycelia were harvested by filtration over two layers of miracloth and washed with sterilized water. Harvested mycelia were then lyophilized and ground in liquid nitrogen. Total RNA was extracted from mycelia of each sample using the RNAiso Reagent (TaKaRa Co., Dalian, China), and $10 \mathrm{mg}$ of each RNA sample was used for reverse transcription with RevertAid H Minus First Strand cDNA Synthesis Kit employing the oligo $(\mathrm{dT})_{18}$ primer (Fermentas Life Sciences, Burlington, ON, Canada). The expression of each gene was determined by quantitative real-time PCR with the primers listed in Table S1. For each sample, amplification of the actin gene was performed by PCR with the FvActin-F + FvActin-R primer pair (Table S1) was performed as a reference. The experiment was repeated on three independent occasions.

\subsection{Western Blotting Assay}

Six mycelial plugs were inoculated into $150 \mathrm{~mL}$ liquid $\mathrm{CM}$ and incubated at $25^{\circ} \mathrm{C}$ in a shaker (200 rpm) for $36 \mathrm{~h}$. Mycelia were harvested, washed with deionized water and ground in liquid nitrogen. Approximately, $200 \mathrm{mg}$ of finely ground mycelia were re-suspended in $1 \mathrm{~mL}$ of extraction buffer [50 mM Tris-HCl, pH 7.5, 100 mM NaCl, 5 mM EDTA (Ethylene Diamine Tetraacetic Acid), 1\% Triton X-100, 2 mM PMSF (Phenylmethanesulfonyl fluoride)] and 10- $\mu \mathrm{L}$ of protease inhibitor cocktail (Sangon, Shanghai, China). After homogenization with a vortex shaker, the lysate was centrifuged at $14,000 \times g$ in a microcentrifuge for $20 \mathrm{~min}$ at $4{ }^{\circ} \mathrm{C}$ [30]. The resulting proteins were separated by SDS polyacrylamide gel electrophoresis (10\% denaturating gel) and transferred to an Immobilon-P transfer membrane (Millipore, Billerica, MA, USA) using electroblotting apparatus (Bio-Rad, Hercules, CA, USA). Phosphor-p44/42 MAP kinase antibody and Phosphor-p38 MAP kinase antibody (Cell Signaling Technology, Boston, MA, USA) were used for detection of phosphorylated FvMgv1 and FvHog1, respectively [13]. GAPDH (glyceraldehyde-3-phosphate dehydrogenase) was detected in samples using anti-GAPDH antibody (Huabio, Hangzhou, China) as a reference. The experiment was repeated on two independent occasions.

\section{Conclusions}

In summary, we genetically and biochemically characterized the functions of FvSet1 in F. verticillioides. The results of the current study demonstrate that FvSet1 plays an important role in FB1 biosynthesis, fungal growth and virulence, and environmental stress responses in F. verticillioides. These results present a relationship between FvSet1 and FB1 biosynthesis in F. verticillioides. However, investigations are still required for elucidating the mechanisms by which H3K4me regulates secondary metabolism.

Supplementary Materials: The following are available online at www.mdpi.com/2072-6651/9/2/43/s1. Table S1: Primers used in this study. Figure S1: FvSet1 is homologous to those counterparts from yeasts and other filamentous fungi. (A) alignments of amino acid sequences of Set1 orthologs from Fusarium verticillioides (FvSet1), Saccharomyces cerevisiae (ScSet1), Schizosaccharomyces pombe (SpSet1), Neurospora crassa (NcSet1), Podospora anserina (PaSet1), Colletotrichum gloeosporioides (CgSet1), Aspergillus nidulans (AnSet1), and Fusarium graminearum (FgSet1). Boxshade program was used to highlight identical (black shading) or similar (grey shading) amino acids; (B) phylogenetic tree generated using the neighbour-joining method with Mega 4.1 software on the basis of the deduced amino acid sequences of FvSet1 from F. verticillioides strain 7600 and those from S. cerevisiae, S. pombe, N. crassa, C. gloeosporioides, P. anserine, A. nidulans, and F. graminearum. The bootstrap values are indicated on the phylogenetic tree. Figure S2: Schematic representation of the FvSET1 disruption strategy and Southern blotting analyses of the deletion mutants. (A) Schematic diagram of the FvSET1 gene, and gene replacement construct; (B) Southern blot analysis of Dra I-digested genomic DNA of the wild type 7600 and $\triangle$ FvSet1 mutant hybridized 
with the FvSET1 gene and HPH probes, respectively. Figure S3: Relative expression of FUM genes in the $\triangle$ FvSet1. The relative expression levels of FUM genes in $\triangle$ FvSet1 are the relative amounts of mRNA of the gene in the wild-type progenitor. The expression level of the actin gene was used as an internal reference for each sample. Line bars in each column denote standard errors of three repeated experiments.

Acknowledgments: This work was supported by the National Natural Science Foundation of China [grant number 0201600593], the Natural Science Foundation of Jiangsu Province, China [grant number BK20160719], and the Fundamental Research Funds for the Central Universities [grant number Y0201600124]. We thank Professor Guodong Lu at Fujian Agriculture and Forestry University and Professor Huiquan Liu at Northwest A\&F University for kindly providing the F. verticillioides wild type 7600.

Author Contributions: Xuewen Gao and Qin Gu conceived and designed the experiments; Qin Gu, Hafiz Abdul Samad Tahir, Huijun Wu, Hao Zhang, Xiao Sun, and Hai Huang performed the experiments; Tiantian Ji, and Liming Wu analyzed the data; Qin Gu and Huijun Wu wrote the manuscript.

Conflicts of Interest: The authors declare no conflict of interest.

\section{References}

1. Munkvold, G.P. Cultural and genetic approaches to managing mycotoxins in maize. Annu. Rev. Phytopathol. 2003, 41, 99-116. [CrossRef] [PubMed]

2. Nelson, P.E. Taxonomy and biology of Fusarium moniliforme. Mycopathologia 1992, 117, 29-36. [CrossRef] [PubMed]

3. Howard, P.C.; Eppley, R.M.; Stack, M.E.; Warbritton, A.; Voss, K.A.; Lorentzen, R.J.; Kovach, R.M.; Bucci, T.J. Fumonisin b1 carcinogenicity in a two-year feeding study using F344 rats and B6C3F1 mice. Environ. Health Perspect. 2001, 109, 277-282. [CrossRef] [PubMed]

4. Park, D.L.; Troxell, T.C. U.S. Perspective on mycotoxin regulatory issues. Adv. Exp. Med. Biol. 2002, 504, 277-285. [PubMed]

5. Glenn, A.E.; Zitomer, N.C.; Zimeri, A.M.; Williams, L.D.; Riley, R.T.; Proctor, R.H. Transformation-mediated complementation of a FUM gene cluster deletion in Fusarium verticillioides restores both fumonisin production and pathogenicity on maize seedlings. Mol. Plant-Microbe Interact. 2008, 21, 87-97. [CrossRef] [PubMed]

6. Alexander, N.J.; Proctor, R.H.; McCormick, S.P. Genes, gene clusters, and biosynthesis of trichothecenes and fumonisins in Fusarium. Toxin Rev. 2009, 28, 198-215. [CrossRef]

7. Yin, S.; Guo, X.; Li, J.; Fan, L.; Hu, H. Fumonisin B1 induces autophagic cell death via activation of ERN1-MAPK8/9/10 pathway in monkey kidney MARC-145 cells. Arch. Toxicol. 2016, 90, 985-996. [CrossRef] [PubMed]

8. Strahl, B.D.; Allis, C.D. The language of covalent histone modifications. Nature 2000, 403, 41-45. [CrossRef] [PubMed]

9. Gu, S.G.; Fire, A. Partitioning the C. elegans genome by nucleosome modification, occupancy, and positioning. Chromosoma 2010, 119, 73-87. [CrossRef] [PubMed]

10. Zhang, X.Y.; Bernatavichute, Y.V.; Cokus, S.; Pellegrini, M.; Jacobsen, S.E. Genome-wide analysis of mono-, di- and trimethylation of histone H3 lysine 4 in Arabidopsis thaliana. Genome Biol. 2009, 10, R62. [CrossRef] [PubMed]

11. Peters, A.H.; Kubicek, S.; Mechtler, K.; O’Sullivan, R.J.; Derijck, A.A.; Perez-Burgos, L.; Kohlmaier, A.; Opravil, S.; Tachibana, M.; Shinkai, Y.; et al. Partitioning and plasticity of repressive histone methylation states in mammalian chromatin. Mol. Cell 2003, 12, 1577-1589. [CrossRef]

12. Yao, T.T.; Cohen, R.E. A cryptic protease couples deubiquitination and degradation by the proteasome. Nature 2002, 419, 403-407. [CrossRef] [PubMed]

13. Liu, Y.; Liu, N.; Yin, Y.; Chen, Y.; Jiang, J.; Ma, Z. Histone H3K4 methylation regulates hyphal growth, secondary metabolism and multiple stress responses in Fusarium graminearum. Environ. Microbiol. 2015, 17, 4615-4630. [CrossRef] [PubMed]

14. Pham, K.T.M.; Mo Inoue, Y.; Vu, B.V.; Nguyen, H.H.; Nakayashiki, T.; Ikeda, K.; Nakayashiki, H. MoSet1 (Histone H3K4 methyltransferase in Magnaporthe oryzae) regulates global gene expression during infection-related morphogenesis. PLoS Genet. 2015, 11, e1005385.

15. Roguev, A.; Schaft, D.; Shevchenko, A.; Pijnappe, W.W.; Wilm, M.; Aasland, R.; Stewart, A.F. The Saccharomyces cerevisiae Set1 complex includes an Ash2 homologue and methylates histone 3 lysine 4. EMBO J. 2001, 20, 7137-7148. [CrossRef] [PubMed] 
16. Krogan, N.J.; Dover, J.; Khorrami, S.; Greenblatt, J.F.; Schneider, J.; Johnston, M.; Shilatifard, A. COMPASS, a histone $\mathrm{H} 3$ (lysine 4) methyltransferase required for telomeric silencing of gene expression. J. Biol. Chem. 2002, 277, 10753-10755. [CrossRef] [PubMed]

17. Schneider, J.; Wood, A.; Lee, J.S.; Schuster, R.; Dueker, J.; Maguire, C.; Swanson, S.K.; Florens, L.; Washburn, M.P.; Shilatifard, A. Molecular regulation of histone H3 trimethylation by COMPASS and the regulation of gene expression. Mol. Cell 2005, 19, 849-856. [CrossRef] [PubMed]

18. Nislow, C.; Ray, E.; Pillus, L. SET1, a yeast member of the Trithorax family, functions in transcriptional silencing and diverse cellular processes. Mol. Biol. Cell 1997, 8, 2421-2436. [CrossRef] [PubMed]

19. Santos-Rosa, H.; Schneider, R.; Bannister, A.J.; Sherriff, J.; Bernstein, B.E.; Emre, N.C.; Schreiber, S.L.; Mellor, J.; Kouzarides, T. Active genes are tri-methylated at K4 of histone H3. Nature 2002, 419, 407-411. [CrossRef] [PubMed]

20. Ng, H.H.; Robert, F.; Young, R.A.; Struhl, K. Targeted recruitment of set1 histone methylase by elongating pol II provides a localized mark and memory of recent transcriptional activity. Mol. Cell 2003, 11, 709-719. [CrossRef]

21. Cheng, H.L.; He, X.Y.; Moore, C. The essential WD repeat protein Swd2 has dual functions in RNA polymerase II transcription termination and lysine 4 methylation of histone H3. Mol. Cell. Biol. 2004, 24, 2932-2943. [CrossRef] [PubMed]

22. Palmer, J.M.; Keller, N.P. Secondary metabolism in fungi: Does chromosomal location matter? Curr. Opin. Microbiol. 2010, 13, 431-436. [CrossRef] [PubMed]

23. Palmer, J.M.; Bok, J.W.; Lee, S.; Dagenais, T.R.; Andes, D.R.; Kontoyiannis, D.P.; Keller, N.P. Loss of CclA, required for histone 3 lysine 4 methylation, decreases growth but increases secondary metabolite production in Aspergillus fumigatus. Peer] 2013, 1, e4. [CrossRef] [PubMed]

24. Noma, K.; Grewal, S.I.S. Histone H3 lysine 4 methylation is mediated by Set1 and promotes maintenance of active chromatin states in fission yeast. Proc. Natl. Acad. Sci. USA 2002, 99, 16438-16445. [CrossRef] [PubMed]

25. Klittich, C.J.R.; Leslie, J.F. Chlorate-Resistant, Nitrate-Utilizing (Crn) Mutants in Fusarium-Moniliforme (Gibberella-Fujikuroi). Phytopathology 1989, 135, 721-727. [CrossRef]

26. Shim, W.B.; Woloshuk, C.P. Regulation of fumonisin B(1) biosynthesis and conidiation in Fusarium verticillioides by a cyclin-like (C-type) gene, FCC1. Appl. Environ. Microbiol. 2001, 67, 1607-1612. [CrossRef] [PubMed]

27. Plattner, R.D.; Desjardins, A.E.; Leslie, J.F.; Nelson, P.E. Identification and characterization of strains of Gibberella fujikuroi mating population A with rare fumonisin production phenotypes. Mycologia 1996, 88, 416-424. [CrossRef]

28. Plattner, R.D.; Weisleder, D.; Poling, S.M. Analytical determination of fumonisins and other metabolites produced by Fusarium moniliforme and related species on corn. Fumonisins Food 1996, 392, 57-64.

29. Gu, Q.; Zhang, C.; Yu, F.; Yin, Y.; Shim, W.B.; Ma, Z. Protein kinase FgSch9 serves as a mediator of the target of rapamycin and high osmolarity glycerol pathways and regulates multiple stress responses and secondary metabolism in Fusarium graminearum. Environ. Microbiol. 2015, 17, 2661-2676. [CrossRef] [PubMed]

30. Gu, Q.; Chen, Y.; Liu, Y.; Zhang, C.; Ma, Z. The transmembrane protein FgSho1 regulates fungal development and pathogenicity via the MAPK module Ste50-Ste11-Ste7 in Fusarium graminearum. New Phytol. 2015, 206, 315-328. [CrossRef] [PubMed]

(C) 2017 by the authors; licensee MDPI, Basel, Switzerland. This article is an open access article distributed under the terms and conditions of the Creative Commons Attribution (CC BY) license (http:/ / creativecommons.org/licenses/by/4.0/). 